PROCEEDINGS OF THE AMERICAN MATHEMATICAL SOCIETY

Volume 127, Number 3, March 1999, Pages 915-924

S 0002-9939(99)04608-0

\title{
A CLASS OF DIFFERENTIABLE TORAL MAPS WHICH ARE TOPOLOGICALLY MIXING
}

\author{
NAOYA SUMI
}

(Communicated by Mary Rees)

\begin{abstract}
We show that on the 2-torus $\mathbb{T}^{2}$ there exists a $C^{1}$ open set $\mathcal{U}$ of $C^{1}$ regular maps such that every map belonging to $\mathcal{U}$ is topologically mixing but is not Anosov. It was shown by Mañé that this property fails for the class of $C^{1}$ toral diffeomorphisms, but that the property does hold for the class of $C^{1}$ diffeomorphisms on the 3 -torus $\mathbb{T}^{3}$. Recently Bonatti and Diaz proved that the second result of Mañé is also true for the class of $C^{1}$ diffeomorphisms on the $n$-torus $\mathbb{T}^{n}(n \geq 4)$.
\end{abstract}

The concept of sensitive dependence on initial conditions has played an important role in the development of the theory of chaotic dynamical systems (see Devaney [4]). We know that this concept contains that of topological mixing, which is well known from the hyperbolic theory. Thus it will be natural to ask how big the class of topological mixing is. We discuss this question on dynamical systems defined by $C^{1}$ regular maps of the 2 -torus.

Let $\mathbb{T}^{n}$ be an $n$-torus and let $\pi: \mathbb{R}^{n} \rightarrow \mathbb{T}^{n}$ be the natural projection. A metric for $\mathbb{T}^{n}$ is defined by $d(x, y)=\inf \left\{|\bar{x}-\bar{y}| \mid \bar{x}, \bar{y} \in \mathbb{R}^{n}, \pi(\bar{x})=x, \pi(\bar{y})=y\right\}\left(x, y \in \mathbb{T}^{n}\right)$ where $|\cdot|$ denotes the euclidean norm of $\mathbb{R}^{n}$. Let $f: \mathbb{T}^{n} \rightarrow \mathbb{T}^{n}$ be a continuous surjection. $f$ has sensitive dependence on initial conditions if there is $\delta>0$ such that for each $x \in \mathbb{T}^{n}$ and each neighborhood $U$ of $x$ there exist $y \in U$ and $n \geq 0$ such that $d\left(f^{n}(x), f^{n}(y)\right)>\delta$. If for nonempty open sets $U, V$ there exists $N>0$ such that $U \cap f^{n}(V) \neq \varnothing$ for all $n \geq N$, then $f: \mathbb{T}^{n} \rightarrow \mathbb{T}^{n}$ is said to be topologically mixing. $f$ is positively expansive if there is a constant $\delta>0$ such that if $x \neq y$, then $d\left(f^{n}(x), f^{n}(y)\right)>\delta$ for some nonnegative integer $n$ ( $\delta$ is called an expansive constant for $f$ ). For continuous surjective maps of the $n$-torus the above properties satisfy the following implications:

$$
\begin{aligned}
\text { positive expansivity } & \Longrightarrow \text { topological mixing } \\
& \Longrightarrow \text { sensitive dependence on initial conditions. }
\end{aligned}
$$

Whenever on the 1-torus $\mathbb{T}^{1}$ a local homeomorphism $f$ is topologically mixing, it is positively expansive. This is easily checked as follows. Let $\delta>0$ be small enough. For $x, y \in \mathbb{T}^{1}$ with $x \neq y$ suppose $d\left(f^{n}(x), f^{n}(y)\right) \leq \delta$ for $n \geq 0$. Then the open interval with length $\leq \delta,(x, y)$, satisfies $f^{n}((x, y)) \subset U_{\delta}\left(f^{n}(x)\right)$ for $n \geq 0$. Since $f$ is topologically mixing, for arbitrary nonempty open sets $U_{1}, U_{2}$ there exists

Received by the editors November 26, 1996 and, in revised form, June 26, 1997.

1991 Mathematics Subject Classification. Primary 58F12.

Key words and phrases. Anosov differentiable map, DA-map, sensitive dependence on initial conditions, topological mixing, transversal homoclinic point. 
$N>0$ such that $f^{n}((x, y)) \cap U_{1} \neq \varnothing$ and $f^{n}((x, y)) \cap U_{2} \neq \varnothing$ for $n \geq N$. But this is inconsistent since $f^{n}((x, y)) \subset U_{\delta}\left(f^{n}(x)\right)$ for $n \geq 0$. Thus, $d\left(f^{n}(x), f^{n}(y)\right)>\delta$ for some $n \geq 0$, that is positively expansive.

Since the positively expansive property cannot hold for homeomorphisms (see $[2]), f$ is not injective. This implies that on the 1 -torus $\mathbb{T}^{1}$ the $C^{1}$-interior of the class of regular maps that are topologically mixing equals the class of expanding maps. On the 2-torus Mañé [7] proved that the $C^{1}$-interior of topologically mixing diffeomorphisms equals the class of Anosov diffeomorphisms, and moreover he proved that there exists a $C^{1}$ open set $\mathcal{U}$ of $C^{1}$ diffeomorphisms on the 3 -torus $\mathbb{T}^{3}$, such that every diffeomorphism belonging to $\mathcal{U}$ is topologically mixing and is not Anosov. Recently Bonatti and Diaz [3] extended to the class of $C^{1}$ diffeomorphisms on the $n$-torus $\mathbb{T}^{n}(n \geq 4)$ the second result obtained by Mañé.

The aim of this paper is to show the following theorem on the class of regular maps.

Theorem A. On the 2-torus $\mathbb{T}^{2}$ the $C^{1}$-interior of regular maps that are topological mixing is bigger than the class of $C^{1}$ Anosov differentiable maps.

Theorem A will be obtained by proving the following

Theorem B. The class of all $C^{1}$ regular maps contains a nonempty $C^{1}$ open set $\mathcal{U}$ such that every regular map belonging to $\mathcal{U}$ is topologically mixing, but is not Anosov.

Palis and Takens posed the following problem in [11]: Are the diffeomorphisms with a hyperbolic limit set $C^{1}$-dense in the space of all surface diffeomorphisms? This is still unsolved. But Theorem B indicates that the answer is no for the space of all $C^{1}$ regular maps on the 2-torus.

Before starting the proof we prepare a few basic notations for completeness. Let $f: \mathbb{T}^{2} \rightarrow \mathbb{T}^{2}$ be a regular map, i.e. a differentiable map such that for every $x \in \mathbb{T}^{2}$ the derivative $D_{x} f: T_{x} \mathbb{T}^{2} \rightarrow T_{f(x)} \mathbb{T}^{2}$ is surjective. By the inverse function theorem, $f$ is a local diffeomorphism. We denote by $\prod_{-\infty}^{\infty} \mathbb{T}^{2}$ the product topological space with the metric $\tilde{d}\left(\left(x_{j}\right),\left(y_{j}\right)\right)=\sum_{-\infty}^{\infty} 2^{-|j|} d\left(x_{j}, y_{j}\right)$ for $\left(x_{j}\right),\left(y_{j}\right) \in \prod_{-\infty}^{\infty} \mathbb{T}^{2}$, and define a natural projection $\tilde{\pi}: \prod_{-\infty}^{\infty} \mathbb{T}^{2} \rightarrow \mathbb{T}^{2}$ by $\tilde{\pi}\left(\left(x_{i}\right)\right)=x_{0}$. For an $f$-invariant set $\Lambda(f(\Lambda)=\Lambda)$ we write $\Lambda_{f}=\left\{\left(x_{j}\right) \mid x_{j} \in \Lambda, f\left(x_{j}\right)=x_{j+1}(j \in \mathbb{Z})\right\}$. Then a homeomorphism $\tilde{f}: \Lambda_{f} \rightarrow \Lambda_{f}$ is defined by $\tilde{f}\left(\left(x_{i}\right)\right)=\left(f\left(x_{i}\right)\right)$ for $\left(x_{i}\right) \in \Lambda_{f}$ and $\tilde{\pi} \circ \tilde{f}=f \circ \tilde{\pi}$ holds. The system $\left(\Lambda_{f}, \tilde{f}\right)$ is said to be the inverse limit system of $(\Lambda, f)$.

For an $f$-invariant closed subset $\Lambda$ if there exist constants $C>0$ and $0<\lambda<1$ such that for $\tilde{x}=\left(x_{j}\right) \in \Lambda_{f}$ the tangent space $T_{x_{0}} \mathbb{T}^{2}$ splits into the direct sum $T_{x_{0}} \mathbb{T}^{2}=E^{s}(\tilde{x}) \oplus E^{u}(\tilde{x})$ of $D f$-invariant subspaces $E^{s}(\tilde{x})$ and $E^{u}(\tilde{x})$ satisfying

$$
\begin{cases}\left|D_{x_{0}} f^{n}(v)\right| \leq C \lambda^{n}|v| & \left(v \in E^{s}(\tilde{x})\right), \\ \left|\left(D_{x_{-n}} f^{n}\right)^{-1}(v)\right| \leq C \lambda^{n}|v| & \left(v \in E^{u}(\tilde{x})\right)\end{cases}
$$

for all $n \geq 0$, then $\Lambda$ is hyperbolic. If $\mathbb{T}^{2}$ is a hyperbolic set of $f$, then $f: \mathbb{T}^{2} \rightarrow$ $\mathbb{T}^{2}$ is called an Anosov differentiable map. If, in particular, $T_{x_{0}} \mathbb{T}^{2}=E^{u}(\tilde{x})$ for all $\tilde{x}=\left(x_{i}\right) \in \mathbb{T}_{f}^{2}$, then $f$ is said to be expanding. Every expanding map is positively expansive. However, in general a positively expansive regular map need not be expanding ([2]). For the properties of Anosov diffeomorphisms, Anosov 
differentiable maps and expanding maps, we refer to Anosov [1], Smale [14], Franks [5], Manning [9], Mañé and Pugh [8], Przytycki [12] and Shub [13].

For a hyperbolic set $\Lambda$ and $\tilde{x}=\left(x_{j}\right) \in \Lambda_{f}$ define

$$
\begin{gathered}
W_{\varepsilon}^{s}(\tilde{x}, f)=\left\{y \in \mathbb{T}^{2} \mid d\left(f^{j}(x), f^{j}(y)\right) \leq \varepsilon(j \geq 0)\right\}, \\
W_{\varepsilon}^{u}(\tilde{x}, f)=\left\{\begin{array}{l|l}
y \in \mathbb{T}^{2} & \begin{array}{c}
\text { there exists } \tilde{y}=\left(y_{j}\right) \in \mathbb{T}_{f}^{2} \text { such that } \\
y=y_{0} \text { and } d\left(x_{j}, y_{j}\right) \leq \varepsilon(j \leq 0)
\end{array}
\end{array}\right\} .
\end{gathered}
$$

Then $W_{\varepsilon}^{\sigma}(\tilde{x}, f)$ is a manifold which is tangent at $x_{0}$ to $E^{\sigma}(\tilde{x})$ for $\sigma=s, u([12])$. The sets $W_{\varepsilon}^{s}(\tilde{x}, f)$ and $W_{\varepsilon}^{u}(\tilde{x}, f)$ are called the local stable manifold and the local unstable manifold respectively.

Let $f: \mathbb{T}^{2} \rightarrow \mathbb{T}^{2}$ be a regular map, and let $p \in \mathbb{T}^{2}$ be a fixed point of $f . p$ is called hyperbolic if $D_{p} f$ has no eigenvalues of absolute value one. A hyperbolic fixed point is the simplest example of a hyperbolic set. We say that $p$ is a sink if all the eigenvalues of $D_{p} f$ are less than one in absolute value and $p$ is a source if all the eigenvalues of $D_{p} f$ are greater than one in absolute value. A hyperbolic fixed point $p$ is a saddle if $p$ is neither a sink nor a source. (The same definition applies to a periodic point of a differentiable map of an arbitrary manifold.)

Let $f: \mathbb{T}^{2} \rightarrow \mathbb{T}^{2}$ be a regular map. Suppose that $f$ has a saddle fixed point $p$. Take and fix $\bar{p} \in \mathbb{R}^{2}$ with $\pi(\bar{p})=p$. Then there exists a lifting $\bar{f}: \mathbb{R}^{2} \rightarrow \mathbb{R}^{2}$ of $f$ such that $\bar{f}(\bar{p})=\bar{p}$. Obviously $\bar{f}$ is a diffeomorphism and $\bar{p}$ is a saddle fixed point of $\bar{f}$. For $\varepsilon>0$ define $W_{\varepsilon}^{s}(\bar{p}, \bar{f})=\left\{\bar{x} \in \mathbb{R}^{2}|| \bar{f}^{n}(\bar{x})-\bar{p} \mid \leq \varepsilon(n \geq 0)\right\}$ and $W_{\varepsilon}^{u}(\bar{p}, \bar{f})=\left\{\bar{x} \in \mathbb{R}^{2}|| \bar{f}^{n}(\bar{x})-\bar{p} \mid \leq \varepsilon(n \leq 0)\right\}$. Take $\varepsilon>0$ small enough. Then $W_{\varepsilon}^{\sigma}(\bar{p}, \bar{f})$ is a $C^{1}$ curve for $\sigma=s, u$, and there exists $0<\bar{\lambda}<1$ satisfying

$$
\begin{cases}\left|\bar{f}^{n}(\bar{x})-\bar{p}\right| \leq \bar{\lambda}^{n}|\bar{x}-\bar{p}| & \left(\bar{x} \in W_{\varepsilon}^{s}(\bar{p}, \bar{f}), n \geq 0\right), \\ \left|\bar{f}^{-n}(\bar{x})-\bar{p}\right| \leq \bar{\lambda}^{n}|\bar{x}-\bar{p}| & \left(\bar{x} \in W_{\varepsilon}^{u}(\bar{p}, \bar{f}), n \geq 0\right)\end{cases}
$$

(see Hirsch and Pugh [6]). Put $\tilde{p}=(\cdots, p, p, p, \cdots) \in \mathbb{T}_{f}^{2}$. Since $\pi\left(W_{\varepsilon}^{\sigma}(\bar{p}, \bar{f})\right)=$ $W_{\varepsilon}^{\sigma}(\tilde{p}, f)$ by definition, obviously $W_{\varepsilon}^{\sigma}(\tilde{p}, f)$ is a $C^{1}$-curve, and

(I) $d\left(f^{n}(x), p\right) \leq \bar{\lambda}^{n} d(x, p) \quad\left(x \in W_{\varepsilon}^{s}(\tilde{p}, f), n \geq 0\right)$,

(II) if $\left(x_{i}\right) \in \mathbb{T}_{f}^{2}$ satisfies $d\left(x_{-n}, p\right) \leq \varepsilon$ for $n \geq 0$, then $d\left(x_{-n}, p\right) \leq \bar{\lambda}^{n} d\left(x_{0}, p\right)$ for $n \geq 0$.

For $\tilde{p} \in \mathbb{T}_{f}^{2}$ define the stable and unstable sets by

$$
\begin{gathered}
W^{s}(\tilde{p}, f)=\left\{x \in \mathbb{T}^{2} \mid \lim _{n \rightarrow \infty} d\left(f^{n}(x), p\right)=0\right\}, \\
W^{u}(\tilde{p}, f)=\left\{\begin{array}{l|l}
x \in \mathbb{T}^{2} & \begin{array}{c}
\text { there exists } \tilde{x}=\left(x_{n}\right) \in \mathbb{T}_{f}^{2} \text { such that } \\
x=x_{0} \text { and } \lim _{n \rightarrow \infty} d\left(x_{-n}, p\right)=0
\end{array}
\end{array}\right\} .
\end{gathered}
$$

Then we have $W^{s}(\tilde{p}, f)=\bigcup_{0}^{\infty} f^{-n}\left(W_{\varepsilon}^{s}(\tilde{p}, f)\right)$ and $W^{u}(\tilde{p}, f)=\bigcup_{0}^{\infty} f^{n}\left(W_{\varepsilon}^{u}(\tilde{p}, f)\right)$. If $f$ is not injective, then $W^{s}(\tilde{p}, f)$ is expressed as a countable union of $C^{1}$ curves, and $W^{u}(\tilde{p}, f)$ is not an immersed manifold. Therefore the stable and unstable sets are not manifolds.

On the other hand, the stable and unstable manifolds at $\bar{p}$ of $\bar{f}$ are defined respectively by $W^{s}(\bar{p}, \bar{f})=\left\{\bar{x} \in \mathbb{R}^{2}\left|\lim _{n \rightarrow \infty}\right| \bar{f}^{n}(\bar{x})-\bar{p} \mid=0\right\}$ and $W^{u}(\bar{p}, \bar{f})=$ 
$\left\{\bar{x} \in \mathbb{R}^{2}\left|\lim _{n \rightarrow-\infty}\right| \bar{f}^{n}(\bar{x})-\bar{p} \mid=0\right\}$. Then it is easily checked that

$$
\begin{aligned}
& W^{s}(\bar{p}, \bar{f})=\bigcup_{0}^{\infty} \bar{f}^{-n}\left(W_{\varepsilon}^{s}(\bar{p}, \bar{f})\right), \quad W^{u}(\bar{p}, \bar{f})=\bigcup_{0}^{\infty} \bar{f}^{n}\left(W_{\varepsilon}^{u}(\bar{p}, \bar{f})\right), \\
& W^{s}(\tilde{p}, f) \supset \pi\left(W^{s}(\bar{p}, \bar{f})\right), \quad W^{u}(\tilde{p}, f)=\pi\left(W^{u}(\bar{p}, \bar{f})\right) .
\end{aligned}
$$

In order to show Theorem B we need the technique of the derived-from-Anosov diffeomorphism by Smale [14] (see Williams [15] for details).

Let $A=\left(\begin{array}{ll}3 & 1 \\ 1 & 1\end{array}\right)$. The eigenvalues of $A$ are $\mu=2+\sqrt{2}, \lambda=2-\sqrt{2}$, so $0<\lambda<1<\mu$. Let $\mathbf{v}^{u}$ and $\mathbf{v}^{s}$ be the eigenvectors for $\mu$ and $\lambda$ respectively with $\left|\mathbf{v}^{u}\right|=\left|\mathbf{v}^{s}\right|=1$. We use coordinates $u_{1} \mathbf{v}^{u}+u_{2} \mathbf{v}^{s}$ on $\mathbb{R}^{2}$ and denote by $E^{\sigma}$ the span of the eigenvector $\mathbf{v}^{\sigma}(\sigma=s, u)$. For $\bar{x} \in \mathbb{R}^{2}$ the tangent spaces $T_{\bar{x}} \mathbb{R}^{2}$ and $T_{\pi(\bar{x})} \mathbb{T}^{2}$ can be identified with $\mathbb{R}^{2}$ in a natural way. Then we can also use $\left(u_{1}, u_{2}\right)$-coordinates on $T_{\bar{x}} \mathbb{R}^{2}$ and $T_{\pi(\bar{x})} \mathbb{T}^{2}$. Note that in the $\left(u_{1}, u_{2}\right)$-coordinates $D_{\bar{x}} \pi=\left(\begin{array}{ll}1 & 0 \\ 0 & 1\end{array}\right): T_{\bar{x}} \mathbb{R}^{2} \rightarrow T_{\pi(\bar{x})} \mathbb{T}^{2}$.

Let $r>0$ be small enough. Then $\left.\pi\right|_{B_{r}(0)}$ is injective where $B_{r}(0)$ is the ball of radius $r$ about 0 . Take a small $r^{\prime}$ with $0<r^{\prime}<r$ and let $\delta(s)$ be a bump function such that $\delta(s)=0(s \geq r)$ and $\delta(s)=1\left(s \leq r^{\prime}\right)$. Define $\bar{\varphi}^{t}(\bar{x})=\left(u_{1}, e^{\delta\left(\left|\left(u_{1}, u_{2}\right)\right|\right) t} u_{2}\right)$ for $\bar{x}=\left(u_{1}, u_{2}\right) \in \mathbb{R}^{2}=E^{u} \oplus E^{s}$ and put

$$
\varphi^{t}(x)= \begin{cases}\pi \circ \bar{\varphi}^{t} \circ\left(\left.\pi\right|_{B_{r}(0)}\right)^{-1}(x) & \left(x \in \pi\left(B_{r}(0)\right)\right), \\ x & \left(x \notin \pi\left(B_{r}(0)\right)\right) .\end{cases}
$$

Then the derivative at $p^{0}=\pi(0)$ is $D_{p^{0}} \varphi^{t}=\left(\begin{array}{ll}1 & 0 \\ 0 & e^{t}\end{array}\right)$ in the $\left(u_{1}, u_{2}\right)$-coordinates.

Let $f_{A}: \mathbb{T}^{2} \rightarrow \mathbb{T}^{2}$ be the toral endomorphism induced by the matrix $A$. Since $0<\lambda<1<\mu, f_{A}$ is an Anosov differentiable map and $p^{0}=\pi(0)$ is a saddle fixed point of $f_{A}$. Define $f=\varphi^{\tau} \circ f_{A}$ for a fixed $\tau$ such that $\mu>e^{\tau} \lambda>1$. Suppose that $-\frac{d \delta}{d s}(s)<1 / s(s \geq 0)$. Then we have that $f$ is regular. The map $f$ is called the DA-regular map. Note that in the $\left(u_{1}, u_{2}\right)$-coordinates the derivative of $f$ at $p^{0}$ is $D_{p^{0}} f=D_{p^{0}} \varphi^{\tau} \circ D_{p^{0}} f_{A}=\left(\begin{array}{cc}1 & 0 \\ 0 & e^{\tau}\end{array}\right) \circ\left(\begin{array}{cc}\mu & 0 \\ 0 & \lambda\end{array}\right)=\left(\begin{array}{cc}\mu & 0 \\ 0 & e^{\tau} \lambda\end{array}\right)$, and thus $p^{0}$ is a source.

Let $\tilde{p}^{0}=\left(\cdots, p^{0}, p^{0}, p^{0}, \cdots\right) \in \mathbb{T}_{f_{A}}^{2}$. Denote by $W_{0}^{s}\left(\tilde{p}^{0}, f_{A}\right)$ the arcwise connected component of $W^{s}\left(\tilde{p}^{0}, f_{A}\right)$ containing $p^{0}$. Then $f\left(W_{0}^{s}\left(\tilde{p}^{0}, f_{A}\right)\right)=W_{0}^{s}\left(\tilde{p}^{0}, f_{A}\right)$. The new map $f$ has three fixed points on $W_{0}^{s}\left(\tilde{p}^{0}, f_{A}\right) \cap B_{r}\left(p^{0}\right), p^{0}$ and two new saddle fixed points $p^{1}$ and $p^{2}$. This fact can be seen to be true because $f$ is a monotonically growing function on $W_{0}^{s}\left(\tilde{p}^{0}, f_{A}\right), f\left(p^{0}\right)=p^{0}$ is a source and outside $B_{r}\left(p^{0}\right)$ the slope of the graph of $f$ on $W_{0}^{s}\left(\tilde{p}^{0}, f_{A}\right)$ is still less than one.

Let $\bar{f}: \mathbb{R}^{2} \rightarrow \mathbb{R}^{2}$ be a lifting of $f$ such that $\bar{f}(0)=0$. Since $f$ has three fixed points $p^{0}, p^{1}, p^{2}$ in $B_{r}\left(p^{0}\right)$ and $f \circ \pi=\pi \circ \bar{f}$ on $B_{r}(0), \bar{f}$ also has three fixed points in $B_{r}(0)$. We denote by $\bar{p}^{i}$ the fixed point of $\bar{f}$ corresponding to $p^{i}(i=0,1,2)$.

For all $x \in \mathbb{T}^{2}$ the derivative $D_{x} f$ is expressed as $D_{x} f=\left(\begin{array}{cc}\mu & 0 \\ c(x) & d(x)\end{array}\right)$ in the $\left(u_{1}, u_{2}\right)$-coordinates, and we have $0<d(x) \leq e^{\tau} \lambda$. Since $f \circ \pi=\pi \circ \bar{f}$ and $D_{\bar{x}} \pi=i d\left(\bar{x} \in \mathbb{R}^{2}\right)$, we have that $D_{\bar{x}} \bar{f}=D_{\pi(\bar{x})} f=\left(\begin{array}{cc}\mu & 0 \\ c(\pi(\bar{x})) & d(\pi(\bar{x}))\end{array}\right)$ for $\bar{x} \in \mathbb{R}^{2}$. Put $C=\max \left\{|c(x)| \mid x \in \mathbb{T}^{2}\right\}$ and $D=\min \left\{d(x) \mid x \in \mathbb{T}^{2}\right\}$, and take $L>0$ such 
that

$$
\mu>e^{\tau} \lambda+C / L
$$

Since $\mu>1$, we may fix $m>0$ satisfying $\mu^{m} /(1+L)>1$ and take $\bar{\mu}$ such that

$$
\mu^{m} /(1+L)>\bar{\mu}>1 \text {. }
$$

Let $\bar{\lambda}$ satisfy $\lambda<\bar{\lambda}<1-8 r$ and choose $\delta>0$ so that

$$
\left\{\begin{array}{l}
1 / \lambda(1+\delta)>1 / \bar{\lambda} \\
\bar{\lambda}<1-8 r \sqrt{1+\delta^{2}}, \\
\mu-\delta\left(\frac{C e^{\tau} \lambda}{D}\right)>e^{\tau} \lambda, \\
\delta<1 / L .
\end{array}\right.
$$

For $\bar{x} \in \mathbb{R}^{2}$ define $T^{u}(\bar{x})=\left\{\left(v_{1}, v_{2}\right) \in E^{u}(\bar{x}) \oplus E^{s}(\bar{x})|| v_{2}|\leq L| v_{1} \mid\right\}$ where $E^{u}(\bar{x})$ and $E^{s}(\bar{x})$ denote the subspaces in $T_{\bar{x}} \mathbb{R}^{2}$ corresponding to $E^{u}$ and $E^{s}$ in $\mathbb{R}^{2}$. Then for $\bar{x} \in \mathbb{R}^{2}$ and $0 \neq v \in T^{u}(\bar{x})$

$$
D_{\bar{x}} \bar{f}(v) \in \operatorname{int} T^{u}(\bar{f}(\bar{x})), \quad\left|D_{\bar{x}} \bar{f}^{m}(v)\right|>\bar{\mu}|v|
$$

where $\operatorname{int}(E)$ denotes the interior of $E$. Indeed, for $\left(v_{1}, v_{2}\right) \in T^{u}(\bar{x})$ put $D_{\bar{x}} \bar{f}\left(v_{1}, v_{2}\right)$ $=\left(v_{1}^{\prime}, v_{2}^{\prime}\right)$, and then

$$
\begin{aligned}
\left|v_{2}^{\prime}\right| & =\left|c(\pi(\bar{x})) v_{1}+d(\pi(\bar{x})) v_{2}\right| \leq C\left|v_{1}\right|+e^{\tau} \lambda\left|v_{2}\right| \\
& \leq\left\{C+L e^{\tau} \lambda\right\}\left|v_{1}\right|<L \mu\left|v_{1}\right|=L\left|v_{1}^{\prime}\right| \quad(\text { by (1)) }
\end{aligned}
$$

and so $\left(v_{1}^{\prime}, v_{2}^{\prime}\right) \in T^{u}(\bar{f}(\bar{x}))$. The second inequality is easily checked by (2). Therefore (4) is proved.

For $\bar{x} \in \mathbb{R}^{2}$ we also define $T^{s}(\bar{x})=\left\{\left(v_{1}, v_{2}\right) \in E^{u}(\bar{x}) \oplus E^{s}(\bar{x})|| v_{1}|\leq \delta| v_{2} \mid\right\}$. Since the derivative $D_{\bar{x}} \bar{f}^{-1}$ of $\bar{f}^{-1}$ is expressed as $D_{\bar{x}} \bar{f}^{-1}=\left(\begin{array}{cc}\mu^{-1} & 0 \\ 0 & \lambda^{-1}\end{array}\right)$ for $\bar{x} \notin B_{r}\left(\mathbb{Z}^{2}\right)=\bigcup_{\mathbf{n} \in \mathbb{Z}^{2}} B_{r}(\mathbf{n})$, by (3) we have

$$
\begin{cases}D_{\bar{x}} \bar{f}^{-1}(v) \in \operatorname{int} T^{s}\left(\bar{f}^{-1}(\bar{x})\right) & \left(\bar{x} \in \mathbb{R}^{2}, 0 \neq v \in T^{s}(\bar{x})\right), \\ \left|D_{\bar{x}} \bar{f}^{-1}(v)\right|>\bar{\lambda}^{-1}|v| & \left(\bar{x} \notin B_{r}\left(\mathbb{Z}^{2}\right), 0 \neq v \in T^{s}(\bar{x})\right)\end{cases}
$$

where $\operatorname{int}(E)$ denotes the interior of $E$. The proof of (5) is similar to that of (4) and so we omit the proof.

Then, by the implicit function theorem we see that there exists a small $C^{1}$ neighborhood $\mathcal{U}$ of $f$ such that each $g$ of $\mathcal{U}$ is regular and

(i) in $B_{r}\left(p^{0}\right)$ there exists a fixed point $p_{g}^{i}$ of $g(i=0,1,2)$, and $p_{g}^{0}$ is a source and $p_{g}^{i}$ is a saddle for $i=1,2$,

(ii) every lifting of $g$ satisfies the properties (4) and (5),

(iii) for $x \in \mathbb{T}^{2}$ there exists $y \in g^{-1}(x)$ such that $B_{1 / 4}(y) \cap B_{r}\left(p^{0}\right)=\varnothing$.

The following Proposition C implies Theorem B.

Proposition C. Under the above notation, for $g \in \mathcal{U}$ and two saddle fixed points $p_{g}^{i}(i=1,2)$ of $g$, one has cl $\left(W^{u}\left(\tilde{p}_{g}^{i}, g\right) \cap W^{s}\left(\tilde{p}_{g}^{i}, g\right)\right)=\mathbb{T}^{2}$ where $\tilde{p}_{g}^{i}=\left(\cdots, p_{g}^{i}, p_{g}^{i}\right.$, $\left.p_{g}^{i}, \cdots\right)$ for $i=1,2$. Here $\operatorname{cl}(E)$ denotes the closure of $E$.

By Proposition $\mathrm{C}$ it is clear that $g \in \mathcal{U}$ is topologically mixing. Since $g \in \mathcal{U}$ has a source $p_{g}^{0}$ and a saddle $p_{g}^{i}$ by (i), obviously $g$ is not Anosov. Moreover we have the following result from Proposition C. 
Theorem D. Under the above notation, every $g$ belonging to $\mathcal{U}$ has a set of saddle periodic points that is dense in $\mathbb{T}^{2}$.

To see Theorem D we need the next Lemma E. Let $h: \mathbb{T}^{2} \rightarrow \mathbb{T}^{2}$ be a $C^{1}$-regular map and $p \in \mathbb{T}^{2}$ a saddle fixed point of $h$. Then $x \in \mathbb{T}^{2}$ is a homoclinic point of $p$ if $x \in W^{s}(\tilde{p}, h) \cap W^{u}(\tilde{p}, h) \backslash\{p\}$ where $\tilde{p}=(\cdots, p, p, p, \cdots)$. We say that $x$ is a transversal homoclinic point if there exists a point $\tilde{x}=\left(x_{i}\right) \in \mathbb{T}_{h}^{2}$ such that $x=x_{0}$ and $d\left(x_{-n}, p\right) \rightarrow 0(n \rightarrow \infty)$, and such that for some $n>0$

$$
\begin{aligned}
& x_{n} \in W_{\varepsilon}^{s}(\tilde{p}, h), \quad x_{-n} \in W_{\varepsilon}^{u}(\tilde{p}, h), \\
& D_{x_{-n}} h^{2 n}\left(T_{x_{-n}} W_{\varepsilon}^{u}(\tilde{p}, h)\right)+T_{x_{n}} W_{\varepsilon}^{s}(\tilde{p}, h)=T_{x_{n}} \mathbb{T}^{2} .
\end{aligned}
$$

From the homoclinic point theorem for diffeomorphisms we have the following

Lemma E. Let $p \in \mathbb{T}^{2}$ be a saddle fixed point of a $C^{1}$-regular map $h$. If $x \in \mathbb{T}^{2}$ is a transversal homoclinic point of $p$, then the point $x$ is approximated by saddle periodic points of $h$.

Since $h$ is a local diffeomorphism, the conclusion of Lemma $\mathrm{E}$ is obtained by the technique described in Newhouse [10]. That for $g \in \mathcal{U}$ every homoclinic point of the saddle fixed point $p_{g}^{i}(i=1,2)$ of $g$ is transverse follows from the proof of Proposition C. Therefore Theorem D is concluded. C.

Now to obtain the main result of this paper we have only to show Proposition

Proof of Proposition C. Let $f: \mathbb{T}^{2} \rightarrow \mathbb{T}^{2}$ be the DA-regular map as above and $\mathcal{U}$ be the $C^{1}$ neighborhood of $f$ as in Proposition C. Let $\bar{f}: \mathbb{R}^{2} \rightarrow \mathbb{R}^{2}$ be the lifting of $f$ taken as above. Fix $g \in \mathcal{U}$ and choose a lifting $\bar{g}: \mathbb{R}^{2} \rightarrow \mathbb{R}^{2}$ of $g$ which is close to $\bar{f}$ under the uniform $C^{1}$ topology. By (i) there exists a fixed point $\bar{p}_{g}^{i} \in B_{r}(0)$ of $\bar{g}(i=0,1,2)$, and $\bar{p}_{g}^{0}$ is a source and $\bar{p}_{g}^{i}$ is a saddle for $i=1,2$.

Let $T^{u}(\bar{x})\left(\bar{x} \in \mathbb{R}^{2}\right)$ and $\bar{\mu}>1$ be as above. By (ii), for $\bar{x} \in \mathbb{R}^{2}$ and $0 \neq v \in T^{u}(\bar{x})$,

$$
D_{\bar{x}} \bar{g}(v) \in T^{u}(\bar{g}(\bar{x})), \quad\left|D_{\bar{x}} \bar{g}^{m}(v)\right|>\bar{\mu}|v| .
$$

Let $I$ be a closed interval and let $\bar{k}: I \rightarrow \mathbb{R}^{2}$ be a $C^{1}$-injection. Suppose that

$$
0 \neq \frac{d \bar{k}}{d t}(t) \in T^{u}(\bar{k}(t)) \quad(t \in I)
$$

and for $a, b \in I$ define $d(\bar{k}(a), \bar{k}(b))=\int_{a}^{b}\left|\frac{d \bar{k}}{d t}(t)\right| d t$ where $d$ depends on $k$ as well as $\bar{k}(a), \bar{k}(b)$. The max is attained at the ends of the interval, so it is clearer to define $\ell(\bar{k}(I))$ as $\int_{I}\left|\frac{d \bar{k}}{d t}(t)\right| d t$. From an easy calculation we have $|\bar{k}(a)-\bar{k}(b)| \leq$ $d(\bar{k}(a), \bar{k}(b)) \leq \sqrt{1+L^{2}}|\bar{k}(a)-\bar{k}(b)|$.

Lemma 1. Let $\bar{g}: \mathbb{R}^{2} \rightarrow \mathbb{R}^{2}, m>0$ and $\bar{\mu}>1$ be as above. If ( 7$)$ holds for a $C^{1}$-injection $\bar{k}: I \rightarrow \mathbb{R}^{2}$, then $\ell\left(\bar{g}^{m} \circ \bar{k}(I)\right) \geq \bar{\mu} \ell(\bar{k}(I))$.

Proof. This is clear from (6).

Let $T^{s}(\bar{x})\left(\bar{x} \in \mathbb{R}^{2}\right)$ and $\bar{\lambda}<1$ be as above. Then by (ii) we have

$$
\begin{cases}D_{\bar{x}} \bar{g}^{-1}(v) \in T^{s}\left(\bar{g}^{-1}(\bar{x})\right) & \left(\bar{x} \in \mathbb{R}^{2}, 0 \neq v \in T^{s}(\bar{x})\right), \\ \left|D_{\bar{x}} \bar{g}^{-1}(v)\right|>\bar{\lambda}^{-1}|v| & \left(\bar{x} \notin B_{r}\left(\mathbb{Z}^{2}\right), 0 \neq v \in T^{s}(\bar{x})\right) .\end{cases}
$$


Suppose that for a $C^{1}$-injection $\bar{c}: I \rightarrow \mathbb{R}^{2}$

$$
0 \neq \frac{d \bar{c}}{d t}(t) \in T^{s}(\bar{c}(t)) \quad(t \in I)
$$

Then by (8) we have $|\bar{c}(a)-\bar{c}(b)| \leq d(\bar{c}(a), \bar{c}(b)) \leq \sqrt{1+\delta^{2}}|\bar{c}(a)-\bar{c}(b)|$.

From (8) we have the following Lemma 2.

Lemma 2. Let $\bar{g}: \mathbb{R}^{2} \rightarrow \mathbb{R}^{2}$ and $\bar{\lambda}<1$ be as above. Suppose that (9) holds for a $C^{1}$-injection $\bar{c}: I \rightarrow \mathbb{R}^{2}$. If $\bar{c}(I) \cap B_{r}\left(\mathbb{Z}^{2}\right)=\varnothing$, then $\ell\left(\bar{g}^{-1} \circ \bar{c}(I)\right) \geq \bar{\lambda}^{-1} \ell(\bar{c}(I))$.

By (3) we can take and fix $\hat{\lambda}$ such that

$$
1>\hat{\lambda}>\bar{\lambda} /\left(1-8 r \sqrt{1+\delta^{2}}\right) .
$$

Then we have the following lemma.

Lemma 3. Let $\bar{g}: \mathbb{R}^{2} \rightarrow \mathbb{R}^{2}$ and $\hat{\lambda}$ be as above. Suppose that (9) holds for a $C^{1}$-injection $\bar{c}: I \rightarrow \mathbb{R}^{2}$. If $\ell(\bar{c}(I)) \geq 1 / 4$, then $\ell\left(\bar{g}^{-1} \circ \bar{c}(I)\right) \geq \hat{\lambda}^{-1} \ell(\bar{c}(I))$.

Proof. Let $I=[a, b]$. If $\bar{c}(I) \cap B_{r}\left(\mathbb{Z}^{2}\right)=\varnothing$, then the assertion is ensured by Lemma 2. Thus it suffices to see the lemma for the case $\bar{c}(I) \cap B_{r}\left(\mathbb{Z}^{2}\right) \neq \varnothing$. Since $\ell(\bar{c}(I))<\infty$, there exists a finite sequence $\left\{\mathbf{n}_{i} \mid 1 \leq i \leq k\right\} \subset \mathbb{Z}^{2}$ such that $\bar{c}(I) \cap B_{r}\left(\mathbf{n}_{i}\right) \neq \varnothing$ and $\bar{c}(I) \cap B_{r}\left(\mathbb{Z}^{2}\right) \subset \bigcup_{1}^{k} B_{r}\left(\mathbf{n}_{i}\right)$.

For the case when $k=1$ Lemma 3 is proved as follows. Put $a_{1}=\min \{t \in$ $\left.I \mid \bar{c}(t) \in B_{r}\left(\mathbf{n}_{1}\right)\right\}, b_{1}=\max \left\{t \in I \mid \bar{c}(t) \in B_{r}\left(\mathbf{n}_{1}\right)\right\}$ and $J=\left[a_{1}, b_{1}\right]$. Then we have $\ell(\bar{c}(J)) \leq 2 r \sqrt{1+\delta^{2}}$ and $\bar{c}(I) \cap B_{r}\left(\mathbf{n}_{1}\right) \subset \bar{c}(J)$. By the assumption $\ell(\bar{c}(I)) \geq 1 / 4$ we have

$$
\begin{aligned}
\ell\left(\bar{g}^{-1} \circ \bar{c}(I)\right) & \geq \bar{\lambda}^{-1} \ell(\bar{c}(I \backslash J)) \quad(\text { by Lemma } 2) \\
& =\bar{\lambda}^{-1}\{1-\ell(\bar{c}(J)) / \ell(\bar{c}(I))\} \ell(\bar{c}(I)) \\
& \geq \bar{\lambda}^{-1}\left\{1-8 r \sqrt{1+\delta^{2}}\right\} \ell(\bar{c}(I)) \\
& \geq \hat{\lambda}^{-1} \ell(\bar{c}(I)) \quad(\text { by }(10)) .
\end{aligned}
$$

For the case when $k \geq 2$, put $a_{i}=\min \left\{t \mid \bar{c}(t) \in B_{r}\left(\mathbf{n}_{i}\right)\right\}$ and $b_{i}=\max \{t \mid \bar{c}(t) \in$ $\left.B_{r}\left(\mathbf{n}_{i}\right)\right\}$. Since $r$ is small enough, $\bar{c}\left(\left[a_{i}, b_{i}\right]\right) \cap \bar{c}\left(\left[a_{j}, b_{j}\right]\right)=\varnothing$ for $i \neq j$. Without loss of generality we suppose that $a \leq a_{1} \leq b_{1}<\cdots<a_{k} \leq b_{k} \leq b$. Then $\bar{c}(I)$ is decomposed into finitely many curves $\left\{\bar{c}\left(\left[a, a_{1}\right]\right), \bar{c}\left(\left[a_{1}, a_{2}\right]\right), \cdots, \bar{c}\left(\left[a_{k-1}, a_{k}\right]\right), \bar{c}\left(\left[a_{k}, b\right]\right)\right\}$. Apply the method as above for each curve. Therefore we have the conclusion.

Let $L>0$ and $\delta>0$ be as above and let $\pi^{\sigma}: E^{u} \oplus E^{s} \rightarrow E^{\sigma}(\sigma=u, s)$ be the natural projection. For $\bar{x} \in \mathbb{R}^{2}$ define $C^{u}(\bar{x})=\left\{\bar{y} \in \mathbb{R}^{2}|| \pi^{s}(\bar{x}-\bar{y}) \mid \leq\right.$ $\left.L\left|\pi^{u}(\bar{x}-\bar{y})\right|\right\}$ and $C^{s}(\bar{x})=\left\{\bar{y} \in \mathbb{R}^{2}|| \pi^{u}(\bar{x}-\bar{y})|\leq \delta| \pi^{s}(\bar{x}-\bar{y}) \mid\right\}$. Since $\delta<1 / L$ by (3), $C^{u}(\bar{x}) \cap C^{s}(\bar{y})$ is compact for $\bar{x}, \bar{y} \in \mathbb{R}^{2}$.

Let $I=[-1,1], I_{-}=[-1,0], I_{+}=[0,1]$ and let $\bar{k}$ and $\bar{c}: I \rightarrow \mathbb{R}^{2}$ be $C^{1}-$ injections. Suppose that (7) and (9) hold for $\bar{k}$ and $\bar{c}$ respectively. Obviously $\bar{k}(I) \subset C^{u}(\bar{k}(t))$ and $\bar{c}(I) \subset C^{s}(\bar{c}(t))$ for $t \in I$. Then the following Lemma 4 is easily checked.

Lemma 4. There exists $K>0$ such that if $C^{1}$ injections $\bar{k}$ and $\bar{c}: I \rightarrow \mathbb{R}^{2}$ satisfy the following conditions:

(a) (7) and (9) hold for $\bar{k}$ and $\bar{c}$ respectively,

(b) $\bar{k}(0), \bar{c}(0) \in B_{1}(0)=\left\{\bar{x} \in \mathbb{R}^{2}|| \bar{x} \mid \leq 1\right\}$, 
(c) $\min \left\{\ell\left(\bar{k}\left(I_{*}\right)\right), \ell\left(\bar{c}\left(I_{*}\right)\right) \mid *= \pm\right\} \geq K$, then $\bar{c}(I) \cap \bar{k}(I) \neq \varnothing$.

Let $\bar{p}^{1}$ and $\bar{p}_{g}^{1}$ be as above. It is enough to give the proof of Proposition $\mathrm{C}$ for $p_{g}^{1}$. We write $\bar{p}=\bar{p}^{1}$ and $\bar{p}_{g}=\bar{p}_{g}^{1}$. Let $E^{\sigma}(\bar{p})$ be the subspace in $T_{\bar{p}} \mathbb{R}^{2}$ corresponding to $E^{\sigma}$ for $\sigma=u, s$. Then $E^{u}(\bar{p})\left(E^{s}(\bar{p})\right)$ is the eigenspace of $D_{\bar{p}} \bar{f}$ corresponding to the eigenvalue with absolute value greater (less) than one by the construction of $\bar{f}$.

We denote by $E_{g}^{u}\left(\bar{p}_{g}\right)\left(E_{g}^{s}\left(\bar{p}_{g}\right)\right)$ the eigenspace of $D_{\bar{p}_{g}} \bar{g}$ corresponding to the eigenvalue with absolute value greater (less) than one. For $\sigma=u, s$ we may assume that $E_{g}^{\sigma}\left(\bar{p}_{g}\right) \subset T^{\sigma}\left(\bar{p}_{g}\right)$ by taking $\mathcal{U}$ sufficiently small if necessary. Then for small $\eta>0, T_{\bar{x}} W_{\eta}^{\sigma}\left(\bar{p}_{g}, \bar{g}\right) \subset T^{\sigma}(\bar{x})\left(\bar{x} \in W_{\eta}^{\sigma}\left(\bar{p}_{g}, \bar{g}\right), \sigma=u, s\right)$. Since $W^{s}\left(\bar{p}_{g}, \bar{g}\right)=$ $\bigcup_{0}^{\infty} \bar{g}^{-n}\left(W_{\eta}^{s}\left(\bar{p}_{g}, \bar{g}\right)\right)$ and $W^{u}\left(\bar{p}_{g}, \bar{g}\right)=\bigcup_{0}^{\infty} \bar{g}^{n}\left(W_{\eta}^{u}\left(\bar{p}_{g}, \bar{g}\right)\right)$, by (6) and (8) we have

$$
T_{\bar{x}} W^{\sigma}\left(\bar{p}_{g}, \bar{g}\right) \subset T^{\sigma}(\bar{x}) \quad\left(\bar{x} \in W^{\sigma}\left(\bar{p}_{g}, \bar{g}\right), \sigma=u, s\right) .
$$

By Lemmas 1, 2 and 3 it is checked that the lengths of two connected components of $W^{u}\left(\bar{p}_{g}, \bar{g}\right) \backslash\left\{\bar{p}_{g}\right\}$ are infinite and that $W^{s}\left(\bar{p}_{g}, \bar{g}\right) \backslash\left\{\bar{p}_{g}\right\}$ has a connected component $W_{0}^{s}\left(\bar{p}_{g}, \bar{g}\right)$ such that the length of $W_{0}^{s}\left(\bar{p}_{g}, \bar{g}\right)$ is infinite.

Lemma 5. Let $\mathcal{U}$ and $\tilde{p}_{g}^{1}(g \in \mathcal{U})$ be as in Proposition $C$. Then $\operatorname{cl}\left(W^{u}\left(\tilde{p}_{g}^{1}, g\right)\right)=\mathbb{T}^{2}$ holds.

Proof. Put $\tilde{p}_{g}=\tilde{p}_{g}^{1}$ and let $x \in \mathbb{T}^{2}$ and $U$ be an arbitrary neighborhood of $x$. Then it suffices to see that $U \cap W^{u}\left(\tilde{p}_{g}, g\right) \neq \varnothing$. Take $\bar{x} \in \mathbb{R}^{2}$ with $\pi(\bar{x})=x$. Let $I, I_{-}$and $I_{+}$be as above and let $\bar{c}: I \rightarrow \mathbb{R}^{2}$ be a $C^{1}$-injection with $\bar{c}(0)=\bar{x}$, $0 \neq \frac{d \bar{c}}{d t}(t) \in T^{s}(\bar{c}(t))$ for $t \in I$ and $\pi \circ \bar{c}(I) \subset U$.

Put $c(t)=\pi \circ \bar{c}(t)$ for $t \in I$. Obviously, $c(0)=x$ and $c(I) \subset U$. Since $g \in \mathcal{U}$, by (iii) there exists an infinite sequence $\left\{x_{-n} \mid n \geq 1\right\}$ in $\mathbb{T}^{2}$ such that $g\left(x_{-1}\right)=x$, $g\left(x_{-n}\right)=x_{-n+1}(n \geq 2)$ and

$$
B_{1 / 4}\left(x_{-n}\right) \cap B_{r}\left(p^{0}\right)=\varnothing
$$

where $p^{0}=\pi(0)$ is the source fixed point of $f$. Define a $C^{1}$-injection $c_{-n}: I \rightarrow \mathbb{T}^{2}$ $(n \geq 1)$ such that $c_{-n}(0)=x_{-n}$ and $g^{n} \circ c_{-n}(t)=c(t)$ for $t \in I$. To obtain Lemma 5 it suffices to show that

$$
c_{-n}(I) \cap W^{u}\left(\tilde{p}_{g}, g\right) \neq \varnothing
$$

for some $n \geq 1$. Indeed, if (12) holds, then we have $\varnothing \neq g^{n}\left(c_{-n}(I) \cap W^{u}\left(\tilde{p}_{g}, g\right)\right) \subset$ $c(I) \cap W^{u}\left(\tilde{p}_{g}, g\right) \subset U \cap W^{u}\left(\tilde{p}_{g}, g\right)$.

To see (12) fix $N \geq 1$ and choose $\bar{x}_{-N} \in B_{1}(0)=\left\{\bar{x} \in \mathbb{R}^{2}|| \bar{x} \mid \leq 1\right\}$ such that $\pi\left(\bar{x}_{-N}\right)=x_{-N}$. Let $\bar{c}_{-N}: I \rightarrow \mathbb{R}^{2}$ be a lifting of $c_{-N}: I \rightarrow \mathbb{T}^{2}$ satisfying $\bar{c}_{-N}(0)=\bar{x}_{-N}$. For large $N \geq 1$ we show that

$$
\bar{c}_{-N}(I) \cap W^{u}\left(\bar{p}_{g}, \bar{g}\right) \neq \varnothing .
$$

Indeed, if (13) is proved, then we have $\varnothing \neq \pi\left(\bar{c}_{-N}(I) \cap W^{u}\left(\bar{p}_{g}, \bar{g}\right)\right) \subset c_{-N}(I) \cap$ $W^{u}\left(\tilde{p}_{g}, g\right)$, from which (12) follows.

Therefore, to obtain the conclusion it is only necessary to see (13). Since $\bar{g}^{N} \circ \bar{c}_{-N}$ and $\bar{c}$ are liftings of $c$, we have $\bar{g}^{N} \circ \bar{c}_{-N}(t)=\bar{c}(t)+\mathbf{n}$ for some $\mathbf{n} \in \mathbb{Z}^{2}$, and so $0 \neq \frac{d\left(\bar{g}^{N} \circ \bar{c}_{-N}\right)}{d t}(t) \in T^{s}\left(\bar{g}^{N} \circ \bar{c}_{-N}(t)\right)(t \in I)$. Then by (8) we have $0 \neq \frac{d\left(\bar{g}^{n} \circ \bar{c}_{-N}\right)}{d t}(t) \in$ $T^{s}\left(\bar{g}^{n} \circ \bar{c}_{-N}(t)\right)$ for $t \in I$ and $0 \leq n \leq N$.

Lemma 3 ensures that

$$
\ell\left(\bar{c}_{-N}\left(I_{*}\right)\right) \geq \hat{\lambda}^{-N} \ell\left(\bar{g}^{N} \circ \bar{c}_{-N}\left(I_{*}\right)\right) \quad(*= \pm) .
$$


Indeed, fix $*= \pm$ and $0 \leq n \leq N-1$. If $\ell\left(\bar{g}^{n} \circ \bar{c}_{-N}\left(I_{*}\right)\right)<1 / 4$ holds, then we have $\bar{g}^{n} \circ \bar{c}_{-N}\left(I_{*}\right) \cap B_{r}\left(\mathbb{Z}^{2}\right) \subset \pi^{-1}\left(B_{1 / 4}\left(x_{n-N}\right) \cap B_{r}\left(p^{0}\right)\right)=\varnothing$ by (11), and by Lemma 2

$$
\ell\left(\bar{g}^{n-1} \circ \bar{c}_{-N}\left(I_{*}\right)\right) \geq \hat{\lambda}^{-1} \ell\left(\bar{g}^{n} \circ \bar{c}_{-N}\left(I_{*}\right)\right) .
$$

If we have $\ell\left(\bar{g}^{n} \circ \bar{c}_{-N}\left(I_{*}\right)\right) \geq 1 / 4$, then Lemma 3 holds. Thus we have (15). Since $n$ is arbitrary in $[0, N-1]$, we have (14).

Since $\bar{g}^{N} \circ \bar{c}_{-N}(t)=\bar{c}(t)+\mathbf{n}$ for $t \in I$, by (14) we have $\ell\left(\bar{c}_{-N}\left(I_{*}\right)\right) \geq \hat{\lambda}^{-N} \ell\left(\bar{c}\left(I_{*}\right)\right)$ for $*= \pm$. Therefore the lengths of the two connected components of $\bar{c}_{-N}(I) \backslash$ $\left\{\bar{x}_{-N}\right\}$ increase when $N \nearrow \infty$.

On the other hand, since the lengths of two connected components of $W^{u}\left(\bar{p}_{g}, \bar{g}\right) \backslash$ $\left\{\bar{p}_{g}\right\}$ are infinite and $T_{\bar{x}} W^{u}\left(\bar{p}_{g}, \bar{g}\right) \subset T^{u}(\bar{x})$ for $\bar{x} \in W^{u}\left(\bar{p}_{g}, \bar{g}\right)$, by Lemma 4 we have (13).

We are now in a position to prove Proposition C. Let $\mathcal{U}$ and $\tilde{p}_{g}^{1}(g \in \mathcal{U})$ be as in Proposition $\mathrm{C}$ and put $\tilde{p}_{g}=\tilde{p}_{g}^{1}$. For $z \in \mathbb{T}^{2}$ and $U$ a neighborhood of $z$ it suffices to show that

$$
U \cap W^{u}\left(\tilde{p}_{g}, g\right) \cap W^{s}\left(\tilde{p}_{g}, g\right) \neq \varnothing .
$$

Since $\operatorname{cl}\left(W^{u}\left(\tilde{p}_{g}, g\right)\right)=\mathbb{T}^{2}$ by Lemma 5 , it is clear that $W^{u}\left(\tilde{p}_{g}, g\right) \cap U \neq \varnothing$, so choose a point $x$ from the set. Since $W^{u}\left(\tilde{p}_{g}, g\right)=\pi\left(W^{u}\left(\bar{p}_{g}, \bar{g}\right)\right)$, there exists $\bar{x} \in W^{u}\left(\bar{p}_{g}, \bar{g}\right)$ such that $x=\pi(\bar{x})$. Let $I, I_{-}$and $I_{+}$be as above. We can find a $C^{1}$-injection $\bar{k}$ : $I \rightarrow W^{u}\left(\bar{p}_{g}, \bar{g}\right)$ such that $\bar{k}(0)=\bar{x}, \pi \circ \bar{k}(I) \subset U$ and for $t \in I, 0 \neq \frac{d \bar{k}}{d t}(t) \in T^{u}(\bar{k}(t))$. Put $k(t)=\pi \circ \bar{k}(t)$ for $t \in I$. Then we have $k(0)=x$ and $k(I) \subset U \cap W^{u}\left(\tilde{p}_{g}, g\right)$.

To show Proposition $\mathrm{C}$ it suffices to see that

$$
g^{n}(k(I)) \cap W^{s}\left(\tilde{p}_{g}, g\right) \neq \varnothing
$$

for some $n \geq 0$. Once (16) is established, we can take $y \in k(I)$ satisfying $g^{n}(y) \in$ $W^{s}\left(\tilde{p}_{g}, g\right)$. Obviously, $y \in W^{s}\left(\tilde{p}_{g}, g\right)$. Since $y \in k(I) \subset W^{u}\left(\tilde{p}_{g}, g\right) \cap U$, we have $y \in U \cap W^{u}\left(\tilde{p}_{g}, g\right) \cap W^{s}\left(\tilde{p}_{g}, g\right) \neq \varnothing$.

To prove (16), fix $M>0$ and put $N=M m$ and $\bar{k}_{N}=\bar{g}^{N} \circ \bar{k}$. By (6) and Lemma 1 we have that for $t \in I, 0 \neq \frac{d \bar{k}_{N}}{d t}(t) \in T^{u}\left(\bar{k}_{N}(t)\right)$ and

$$
\ell\left(\bar{k}_{N}\left(I_{*}\right)\right) \geq \bar{\mu}^{M} \ell\left(\bar{k}\left(I_{*}\right)\right) \quad(*= \pm) .
$$

Put $B_{1}(0)=\left\{\bar{x} \in \mathbb{R}^{2}|| \bar{x} \mid \leq 1\right\}$. Then $\bar{x}_{N}=\bar{k}_{N}(0)+\mathbf{n}_{1} \in B_{1}(0)$ for some $\mathbf{n}_{1} \in \mathbb{Z}^{2}$. By (17) the lengths of the two connected components of $\left(\bar{k}_{N}(I)+\mathbf{n}_{1}\right) \backslash\left\{\bar{x}_{N}\right\}$ increase when $N \nearrow \infty$.

On the other hand, let $W_{0}^{s}\left(\bar{p}_{g}, \bar{g}\right)$ be as above. Since the length of $W_{0}^{s}\left(\bar{p}_{g}, \bar{g}\right)$ is infinite and $T_{\bar{y}} W_{0}^{s}\left(\bar{p}_{g}, \bar{g}\right) \subset T^{s}(\bar{y})$ for $\bar{y} \in W_{0}^{s}\left(\bar{p}_{g}, \bar{g}\right)$, there exists a $C^{1}$ injection $\bar{c}$ : $I \rightarrow W_{0}^{s}\left(\bar{p}_{g}, \bar{g}\right)$ such that the lengths of the two connected components of $\bar{c}(I) \backslash\{\bar{c}(0)\}$ are large enough and $0 \neq \frac{d \bar{c}}{d t}(t) \in T^{s}(\bar{c}(t))(t \in I)$. Choose $\mathbf{n}_{2} \in \mathbb{Z}^{2}$ such that $\bar{c}(0)+\mathbf{n}_{2} \in B_{1}(0)$. Then we have $\left(\bar{k}_{N}(I)+\mathbf{n}_{1}\right) \cap\left(\bar{c}(I)+\mathbf{n}_{2}\right) \neq \varnothing$ by Lemma 4 . Therefore,

$$
\begin{aligned}
& \varnothing \neq \pi\left\{\left(\bar{k}_{N}(I)+\mathbf{n}_{1}\right) \cap\left(\bar{c}(I)+\mathbf{n}_{2}\right)\right\} \subset \pi\left\{\left(\bar{k}_{N}(I)+\mathbf{n}_{1}\right) \cap\left(W_{0}^{s}\left(\bar{p}_{g}, \bar{g}\right)+\mathbf{n}_{2}\right)\right\} \\
& \quad \subset \pi\left(\bar{k}_{N}(I)\right) \cap \pi\left(W_{0}^{s}\left(\bar{p}_{g}, \bar{g}\right)\right) \subset g^{N}(k(I)) \cap W^{s}\left(\tilde{p}_{g}, g\right) .
\end{aligned}
$$




\section{REFERENCES}

[1] D.V. Anosov, Geodesic flows on closed Riemannian manifolds with negative curvature, Proc. Steklov Inst. Math. 90 (1967). MR 39:3527

[2] N. Aoki and K. Hiraide, Topological Theory of Dynamical Systems, Mathematical Library, North Holland, 1994. MR 95m:58095

[3] C. Bonatti and L.J. Diaz, Persistent nonhyperbolic transitive diffeomorphisms, Ann. of Math. (2) 143 (1996), 357-396. MR 97d:58122

[4] R.L. Devaney, An Introduction to Chaotic Dynamical Systems, Second Edition, AddisonWesley, 1989. MR 91a:58114

[5] J. Franks, Anosov diffeomorphisms, Global Analysis, Proc. Sympos. Pure Math., 14, Amer. Math. Soc. (1970), 61-93. MR 42:6871

[6] M. Hirsch and C. Pugh, Stable manifolds and hyperbolic sets, Global Analysis, Proc. Sympos. Pure Math., 14, Amer. Math. Soc. (1970), 133-163. MR 42:6872

[7] R. Mañé, Contributions to the stability conjecture, Topology 17 (1978), 383-396. MR 84b:58061

[8] R. Mañé and C. Pugh, Stability of endomorphisms, Lecture Notes in Math., 468, SpringerVerlag (1975), 175-184. MR 58:31264

[9] A. Manning, There are no new Anosov diffeomorphisms on tori, Amer. J. Math. 96 (1974), 422-429. MR 58:11324

[10] S. Newhouse, Hyperbolic limit sets, Trans. Amer. Math. Soc. 167 (1972), 125-150. MR 45:4454

[11] J. Palis and F. Takens, Hyperbolicity and Sensitive Chaotic Dynamics at Homoclinic Bifurcations, Cambridge University Press, 1993. MR 94h:58129

[12] F. Przytycki, Anosov endomorphisms, Studia Math. 58 (1976), 249-285. MR 56:3893

[13] M. Shub, Endomorphisms of compact differentiable manifolds, Amer. J. Math. 91 (1969), 175-199. MR 39:2169

[14] S. Smale, Differentiable dynamical systems, Bull. Amer. Math. Soc. 73 (1967), 747-817. MR 37:3598

[15] R. Williams, The "DA" maps of Smale and structural stability, Global Analysis, Proc. Sympos. Pure Math. 14, Amer. Math. Soc. (1970), 329-334.

Department of Mathematics, Tokyo Metropolitan University, Tokyo 192-03, Japan

E-mail address: sumi@math.metro-u.ac.jp 